\title{
In situ assessment and minimization of nonlinear propagation effects for femtosecond-laser waveguide writing in dielectrics
}

\author{
Andrés Ferrer, ${ }^{1}$ Alexandro Ruiz de la Cruz, ${ }^{1}$ Daniel Puerto, ${ }^{1}$ Wojciech Gawelda, ${ }^{1}$ Juan A. Vallés, ${ }^{2}$ Miguel A. Rebolledo, ${ }^{2}$ \\ Víctor Berdejo, ${ }^{2}$ Jan Siegel, ${ }^{1}$ and Javier Solis ${ }^{1, *}$ \\ ${ }^{1}$ Laser Processing Group, Instituto de Optica, CSIC, C/Serrano 121, 28006 Madrid, Spain \\ ${ }^{2}$ Departamento de Física Aplicada-I3A, Facultad de Ciencias, Universidad de Zaragoza, C/Pedro Cerbuna 12, \\ 50009 Zaragoza, Spain \\ *Corresponding author: j.solis@io.cfmac.csic.es
}

Received May 13, 2010; revised June 28, 2010; accepted June 28, 2010; posted June 29, 2010 (Doc. ID 128417); published July 30, 2010

\begin{abstract}
The effect of nonlinear propagation on the shape of the focal volume has been assessed by in situ plasma emission imaging during the subsurface processing of a commercial phosphate glass. The sample was processed with an elliptically shaped femtosecond-laser beam at $1 \mathrm{kHz}$ repetition rate and scanned transversely with respect to the writing beam axis. As a consequence, optimal conditions for minimizing undesirable nonlinear propagation effects during the production of optical waveguides by direct laser writing have been determined. Under these conditions, it is possible to induce structural transformations and still preserve the focal volume shape associated with the linear propagation regime. While at low pulse energy a single scan laser-written structure does not support a guided mode, the use of multiple scans with minimized nonlinear propagation effects enables the production of optical waveguides. The latter show a significantly improved performance in terms of the refractive index change and propagation losses when compared to single scan waveguides. (C) 2010 Optical Society of America

OCIS codes: $230.7370,190.7110,130.3120,160.4330,230.3120,320.2250$.
\end{abstract}

\section{INTRODUCTION}

Nonlinear processing of dielectrics with femtosecondlaser pulses has opened new routes for the production of functional structures for applications in areas like integrated optics [1,2] or microfluidics [3,4]. In both cases, the laser writing process is based on high peak power densities that can be achieved when a femtosecond-laser pulse is focused inside a dielectric, leading to nonlinear absorption and avalanche ionization [5] in the focal volume and its nearby region, where structural changes occur. When the latter is accompanied by a local increase in the refractive index, optical waveguides can be produced by translating the sample transversally with respect to the optical beam axis [6]. This approach has been successfully applied to the production of various integrated photonic components such as splitters, amplifiers, and lasers [7-10].

From the point of view of laser sources used, femtosecond-laser subsurface structuring can be divided into two processing regimes: high repetition rate $(\mathrm{HRR})$ and low repetition rate (LRR). In the HRR regime, low energy pulses at repetition rates typically of hundreds of kilohertz are used, and the material modification is affected by heat accumulation and its later diffusion, favoring the production of structures with a circular cross section $[11,12]$. In the LRR regime (typically a few kilohertz pulse repetition rates), modification of a material is induced individually by each single pulse since there is sufficient time for the material to recover its initial temperature between consecutive pulses. In addition, the required pulse energy (peak power density) is much higher as compared with the HRR regime, which favors the presence of undesirable nonlinear propagation (NLP) effects.

NLP [13], together with spherical aberration (SA) $[14,15]$, contributes to the distortion of the shape of the focal volume, leading to elongated modification profiles and a reduced efficiency of energy deposition. While it has been shown that the axial elongation of laser-written structures can be overcome by the use of beam shaping strategies (i.e., astigmatic [16] or astigmatic elliptical beam shaping $[17,18]$ ), the control of NLP effects is more complex since it is affected by both material (i.e., nonlinear refractive index) and processing (i.e., pulse peak power, pulse duration, beam polarization, processing depth, beam spatial profile, etc.) parameters. In a recent work [13] we have shown that femtosecond-resolved microscopy can assess the impact of NLP on the distortion of the focal volume shape. That work also identified prefocal depletion [19] and multiple beam filamentation [20] as important nonlinear energy loss channels, preventing efficient subsurface processing.

In the present work we have used plasma emission imaging for in situ assessment of the focal volume shape during femtosecond-laser processing, minimizing efficiently NLP effects and optimizing the performance of waveguides written in a commercial phosphate glass. It is shown that it is feasible to induce structural modifications above the material modification threshold and still preserve the focal volume shape associated with the linear propagation regime. In order to improve the perfor- 
mance of waveguides produced with minimized NLP effects, a multi-scan procedure has been used, enabling the production of optical waveguides with a strongly improved performance in terms of both the refractive index change $(\Delta n)$ and propagation losses, when compared to single scan waveguides. When compared to the multiscan scan approach, first reported by Nasu et al. [21] and subsequently used in the fabrication of a waveguide amplifier [22], it is worth noting that in these works the scans were repeated with a small spatial displacement in order to generate a refractive index modified region with a size/shape adequate for supporting a guided mode. In the present work, we show the advantages of re-scanning exactly over the same region.

\section{EXPERIMENTAL DETAILS}

The layout of the experiment is shown in Fig. 1. A commercial Ti:Sapphire oscillator-amplifier system delivering $100 \mathrm{fs}$ pulses at $1 \mathrm{kHz}$ repetition rate and $1 \mathrm{~W}$ of maximum average power is used for irradiating the sample. The pulse duration can be increased by adjusting the compressor stage in the amplifier system, leading to longer and positively chirped pulses. The sample is a commercial Er-Yb co-doped phosphate glass (2.0 and $2.1 \mathrm{wt}$. \%, respectively, MM2, Kigre Inc.). It is mounted in a motorized stage that allows translating it along either the $x$ - or $y$-axis, at a constant scanning speed of $100 \mu \mathrm{m} / \mathrm{s}$. A $10 \times$, long working distance $(30.5 \mathrm{~mm})$, and low numerical aperture (NA, 0.26) microscope objective (M.O.1) is used to focus the writing beam inside the sample. A $350 \mu \mathrm{m}$ width slit is placed before M.O.1 in order to generate an elliptical beam and thus a disk-shaped focal volume, as first reported by Cheng et al. [23] (see also [17,18]). A $\lambda / 4$ wave plate polarizes the beam circularly so as to reduce propagation losses [24] and to minimize the appearance of periodic nanostructures $[25,26]$. A processing depth of $1.56 \mathrm{~mm}$ was used because NLP effects are clearly observable for sufficiently high pulse energies, while SA effects can still be compensated by the combination of low NA op-

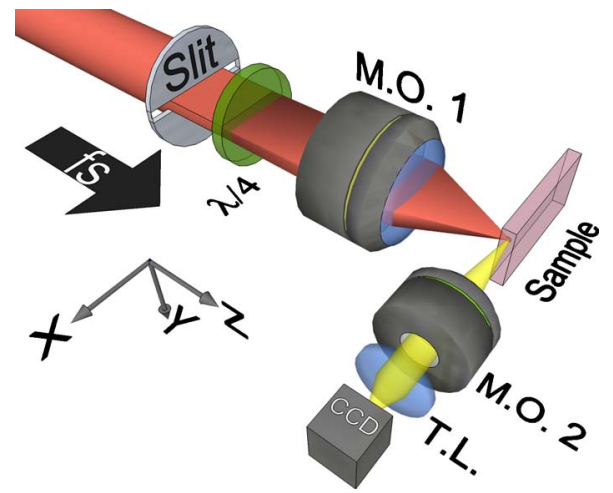

Fig. 1. (Color online) Scheme of the experimental setup used for plasma emission imaging during subsurface processing. The femtosecond-laser beam passes through a slit and is focused inside the sample by a microscope objective (M.O.1) while the sample moves at a transversal scanning speed of $100 \mu \mathrm{m} / \mathrm{s}$. The plasma emission in the focal region is imaged by a lateral imaging system consisting of another microscope objective (M.O.2), a tube lens (T.L.), and a CCD camera. tics and slit beam shaping (see $[17,27]$ for further details regarding the writing setup).

The experimental setup is completed with a $0.42 \mathrm{NA}$ microscope objective, a tube lens, and a 12 bit chargecoupled device (CCD) camera used to image the plasma emission in the focal region. The plasma images were acquired while translating the sample $(100 \mu \mathrm{m} / \mathrm{s}$ speed $)$ transversally to both the irradiation beam and the optical imaging system axis, in order to maintain the image focus on the plasma emission region and to avoid cumulative damage effects. This configuration enables the generation of a local power density and a total dose similar to the one used during the waveguide writing procedure. The plasma images have been normalized to the CCD exposure time.

\section{RESULTS AND DISCUSSION}

Figure 2 shows several plasma emission images obtained for different pulse energies and pulse durations. The shape of the plasma indicates that absorption occurs well before the position of the linear focus of the beam (prefocal depletion [13,19]). For the shortest pulse duration [Fig. 2(a1)], the plasma emission region shows very clearly an arrowhead shape, oriented toward the input sample surface.

This shape can be intuitively understood in terms of self-focusing effects. Since the beam has been shaped with a slit, its spatial profile presents a major axis (i.e., $x$-axis) and a minor axis ( $y$-axis, typically around 20 times smaller than the $x$-axis in the present work) leading to two very different NAs. Both components of the beam experience very different degrees of nonlinear self-focusing on what could be seen as a sort of nonlinear astigmatism. In a complementary simplified picture, the linear focal region has the shape of a flat disk that will be distorted by nonlinear self-focusing: the inner regions, with higher irradiance, will be pulled toward the surface more than the outer ones, leading to the observed plasma shape. These intuitive pictures get distorted by the appearance of other effects (self-phase modulation [28,29] and plasma defocusing [30] that can lead to the appearance of multiple waists and complex spatial structures in the focal region) and that are more relevant the higher is the pulse peak

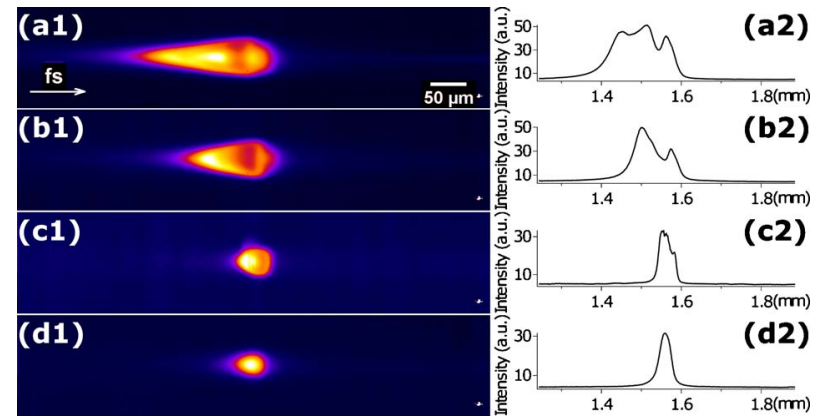

Fig. 2. (Color online) (a1),(b1),(c1) Plasma emission images obtained for pulses of energies of $9.6 \mu \mathrm{J}$ and pulse durations of (a) 100 , (b) 250 , and (c) $550 \mathrm{fs}$. In (d), the pulse duration is $250 \mathrm{fs}$ and the pulse energy is $4 \mu \mathrm{J}$. (a2),(b2),(c2),(d2) The corresponding intensity cross sections along the $z$-axis are shown in the right hand side of the image. 
power as shown in Figs. 2(a2), 2(b2), and 2(c2) with the appearance of multiple peaks.

In Fig. 2(a1), the large extension of plasma emission region confirms that absorption of laser energy occurs over a region much broader than that corresponding to the linear focus of M.O.1. For every pulse energy and processing depth there exists a threshold pulse duration above which NLP becomes negligible, and thus the absorption is limited to the linear focal volume region. However, under these circumstances guiding is not always achievable as it will be shown in Fig. 3 .

For comparison, a plasma emission image corresponding to optimized writing conditions ( $4 \mu \mathrm{J} /$ pulse, pulse duration $\approx 250 \mathrm{fs}$ ) is shown in Fig. $2(\mathrm{~d} 1)$, where the minimization of NLP has been achieved as shown by a nearly Gaussian intensity profile in Fig. 2(d2). Thus, we can assume that under these conditions the position of plasma intensity distribution is the closest to an ideal linear focus (ILF) achievable in our setup. In Figs. 2(a)-2(c), it can be seen how the plasma distribution approaches the ILF as NLP effects are reduced by decreasing the pulse peak power (increasing the pulse duration). Nonlinear absorption prior to the ILF (pre-focal depletion [19]) reduces the number of photons that finally reach the linear focus region, decreasing the global energy coupling process in the ILF as indicated in [13].

Based on the previous assumption, we have estimated the efficiency $(\eta)$ of energy coupling in the ILF in the presence of NLP effects. For that purpose we have used the corresponding overlap integrals,

$$
\eta=\frac{\iint I_{0}(y, z) I_{i}(y, z) d y d z}{\left[\iint\left(I_{0}(y, z)\right)^{2} d y d z \iint\left(I_{i}(y, z)\right)^{2} d y d z\right]^{1 / 2}},
$$

where $I_{0}(y, z)$ is the plasma emission intensity distribution corresponding to the ILF [Fig. 2(d1)] and $I_{i}(y, z)$ are the corresponding intensity distributions for NLPaffected plasma emission images [Figs. 2(a1), 2(b1), and 2(c1)].

The value of $\eta$ (Table 1 ) allows one to compare the energy coupling efficiency, not in terms of the total amount of coupled energy, but of the shape of the energy deposition region when compared to an ideal case. As expected, $\eta$ increases with the pulse duration since the longer is the pulse (that is, the lower is the peak power and the less are

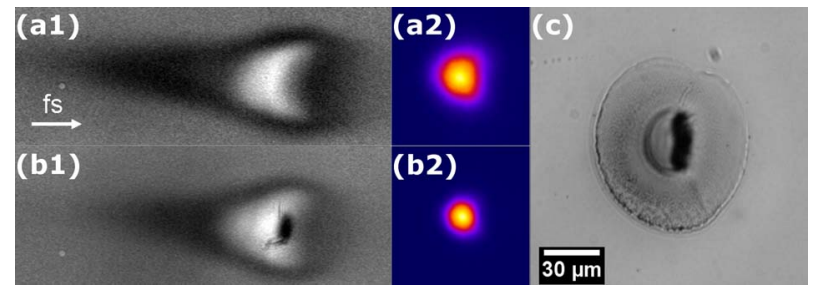

Fig. 3. (Color online) White light microscopy transmission images of waveguides produced with $9.6 \mu \mathrm{J}$ pulses of durations of (a1) 100, (b1) 250, and (c) 550 fs. (a2) and (b2) are the near-field images of the corresponding guided modes at $1550 \mathrm{~nm}$ of the waveguides shown in (a1) and (b1), respectively. The scale is the same in all the images.
Table 1. Energy Coupling Efficiency Parameter ( $\boldsymbol{\eta}$ ) Calculated According to Eq. (1) for the Images Shown in Figs. 2(a1), 2(b1), and 2(c1)

\begin{tabular}{ccc}
\hline $\begin{array}{c}\text { Pulse Duration } \\
(\mathrm{fs})\end{array}$ & Figure & $\eta$ \\
\hline 100 & $2(\mathrm{a} 1)$ & 0.18 \\
250 & $2(\mathrm{~b} 1)$ & 0.22 \\
550 & $2(\mathrm{c} 1)$ & 0.81 \\
\hline
\end{tabular}

NLP effects), the more the shape of the energy deposition region in the material resembles the ILF case.

White light optical microscopy and near-field guided mode images at $1550 \mathrm{~nm}$ corresponding to waveguides written with the conditions of Figs. 2(a)-2(c) are shown in Fig. 3. As expected from the plasma emission images, the degree of structural modification increases with the pulse duration due to larger amount of energy coupled in the focal region. For the shortest pulse duration [Fig. 3(a1)], the white light microscopy image shows a very elongated dark contrasted region (tail), caused by nonlinear absorption in the pre-focal region that results in a negative $\Delta n$ region. This tail spreads over more than $100 \mu \mathrm{m}$ prior to the ILF. Closer to the focal region, a white contrasted area indicates that light guiding occurs, as confirmed by the corresponding near-field image of the guided mode [Fig. 3(a2)] of relatively large size diameter (i.e., $19 \mu \mathrm{m}$ full width at half-maximum), which indicates a poor light confinement. When the pulse length is increased to $250 \mathrm{fs}$ [Figs. 3(b1) and 3(b2)], the dark tail gets shortened and the absorption of pulse energy over a smaller region leads to material damage (in the form of a clearly visible crack inside the light-guiding zone). In spite of damage, the structure supports a guided mode smaller than that written with the shortest pulse. Indeed, refractive index change estimates performed by measuring the effective NA of the waveguides [31] provide values that range from $\Delta n \approx 5 \times 10^{-4}$ (for $100 \mathrm{fs}$ pulses) to $\Delta n \approx 1 \times 10^{-3}$ (for $250 \mathrm{fs}$ pulses). This increase in the refractive index change can be also inferred by comparing the sizes of the guided modes in Figs. 3(a2) and 3(b2). For the longest pulse duration [550 fs, Fig. 3(c)], the decrease in NLP with the pulse duration leads to permanent damage accompanied by surface chipping after polishing, which disables light guiding in the modified zone.

In order to minimize NLP effects without generating such a damage, an alternative strategy for the production of efficient optical waveguides was used. Instead of increasing the pulse duration to reduce the peak power of the laser pulse, we fixed the pulse duration to $250 \mathrm{fs}$ and then decreased the pulse energy down to $4 \mu \mathrm{J}$, for which NLP effects are minimized as shown in Fig. 2(d). For these parameters, the refractive index modified region shows a circular shape [Fig. 4(b1)] although it does not support a guided mode at $1550 \mathrm{~nm}$. In order to increase the refractive index change in the modified region, the sample was scanned several times, which enabled guiding at $1550 \mathrm{~nm}$.

The plot in Fig. 4(a) shows the evolutions of the refractive index change $(\Delta n)$ and the propagation losses of waveguides as functions of the number of scans. Figure 4 

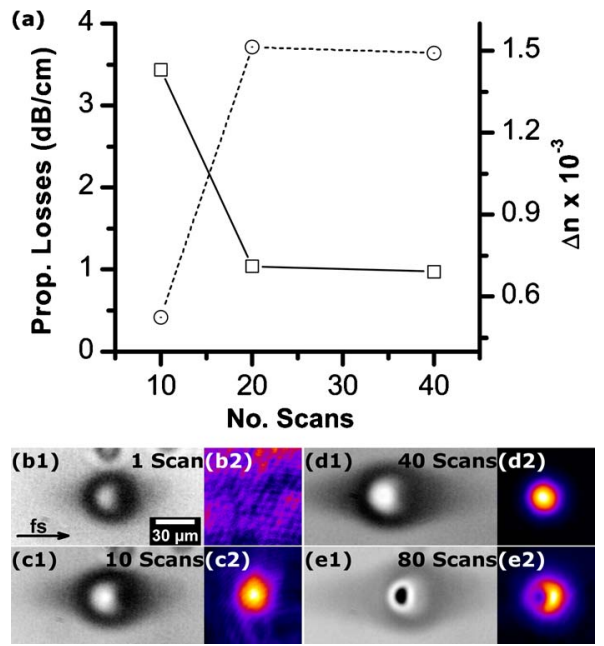

Fig. 4. (Color online) (a) Refractive index change ( $\Delta n$, dashed line with circles) and upper bound for propagation losses (solid line with squares) at $1440 \mathrm{~nm}$ for waveguides written with $4 \mu \mathrm{J}$ and $250 \mathrm{fs}$ laser pulses as functions of the number of scans. (b) (e) White light transmission microscopy and near-field guided mode images at $1550 \mathrm{~nm}$ corresponding to the waveguides in (a). The number of scans is indicated in the images.

also includes white light transmission microscopy images [Figs. 4(b1), 4(c1), 4(d1), and 4(e1)] and near-field guided mode images [Figs. 4(b2), 4(c2), 4(d2), and 4(e2)] of waveguides obtained after 1, 10, 40, and 80 scans. It is remarkable that the transformed region always presents an excellent circular symmetry (consistent with the plasma emission image) unlike the waveguides affected by NLP (Fig. 3). Insertion losses were determined by measuring the output power of the waveguide coupled to a standard telecom fiber and comparing this value with the output power of the fiber itself at $1440 \mathrm{~nm}$, in order to avoid $\mathrm{Er}^{3+}$ ion absorption at $1550 \mathrm{~nm}$. Coupling losses were then estimated by calculating the spatial overlap integrals of the guided mode of the fiber and that of the waveguide, according to [32]. In this way, by subtracting the coupling losses from the total insertion loss, and once Fresnel losses are computed, an upper limit of the propagation loss is obtained, as no extrinsic losses are taken into account such as longitudinal, transversal, or angular displacements.

The refractive index change plot shows how $\Delta n$ increases rapidly with the number of scans, reaching a saturation value around $1.5 \times 10^{-3}$. On the other hand, the propagation losses show the opposite behavior: they quickly decrease with the number of scans, reaching a lower limit below $1 \mathrm{~dB} / \mathrm{cm}$. This decrease in the propagation losses may be due to a smoothing in the sidewall roughness of the waveguides after the repeated scans. Under these writing conditions, the optimal number of scans found is around 40 since for larger values [i.e., 80 scans as shown in Fig. 4(e)] a dark central region appears, likely due to the cumulative defect formation/damage effects.

In summary, it has been shown how NLP effects affect the energy deposition efficiency in laser processing of transparent dielectric materials with femtosecond-laser pulses. By reducing the NLP contribution, the energy coupling efficiency increases, leading to a corresponding in- crease in $\Delta n$ (up to a factor of 2 when increasing the pulse duration to $250 \mathrm{fs}$ ). The minimization of NLP effects also improves the spatial profile of the focal region. Still, in order to reduce the pulse peak power, a further reduction in the pulse energy is required. Under these conditions, the use of a multiple scanning strategy (over the same spatial region) greatly improves the light-guiding performance of written structures. Propagation losses below $1 \mathrm{~dB} / \mathrm{cm}$ were achieved in such structures.

\section{ACKNOWLEDGMENTS}

This work was partially supported by the University of Zaragoza under Project 223/88 and by the Spanish Ministry of Science and Innovation under TEC2008-01183 project. A. Ruiz de la Cruz and W. Gawelda acknowledge their I3P-CSIC postdoctoral contracts (co-funded by the European Social Fund). D. Puerto and A. Ferrer acknowledge their grants under Projects TEC 2005-00074 and TEC 2006-04538.

\section{REFERENCES}

1. K. Hirao, T. Mitsuyu, J. Si, and J. Qiu, Active Glass For Photonic Devices: Photoinduced Structures and Their Application (Springer Verlag, 2001).

2. H. Misawa and S. Juodkazis, 3D Laser Microfabrication: Principles and Applications (Wiley-VCH, 2006).

3. K. Sugioka, Y. Cheng, and K. Midorikawa, "Threedimensional micromachining of glass using femtosecond laser for lab-on-a-chip device manufacture,” Appl. Phys. A 81, 1-10 (2005).

4. R. M. Vazquez, R. Osellame, D. Nolli, C. Dongre, H. van den Vlekkert, R. Ramponi, M. Pollnau, and G. Cerullo, "Integration of femtosecond laser written optical waveguides in a lab-on-chip," Lab Chip 9, 91-96 (2009).

5. B. Stuart, M. Feit, A. Rubenchik, B. Shore, and M. Perry, "Laser-induced damage in dielectrics with nanosecond to subpicosecond pulses," Phys. Rev. Lett. 74, 2248-2251 (1995).

6. K. M. Davis, K. Miura, N. Sugimoto, and K. Hirao, "Writing waveguides in glass with a femtosecond laser," Opt. Lett. 21, 1729-1731 (1996).

7. K. Minoshima, A. M. Kowalevicz, I. Hartl, E. P. Ippen, and J. G. Fujimoto, "Photonic device fabrication in glass by use of nonlinear materials processing with a femtosecond laser oscillator," Opt. Lett. 26, 1516-1518 (2001).

8. R. Osellame, G. Della Valle, N. Chiodo, S. Taccheo, P. Laporta, O. Svelto, and G. Cerullo, "Lasing in femtosecond laser written optical waveguides," Appl. Phys. A 93, 17-26 (2008).

9. G. Della Valle, R. Osellame, and P. Laporta, "Micromachining of photonic devices by femtosecond laser pulses," J. Opt. A, Pure Appl. Opt. 11, 013001 (2009).

10. M. Ams, G. D. Marshall, P. Dekker, M. Dubov, V. Mezentsev, I. Bennion, and M. J. Withford, "Investigation of ultrafast laser-photonic material interactions: challenges for directly written glass photonics," IEEE J. Sel. Top. Quantum Electron. 14, 1370-1381 (2008).

11. C. B. Schaffer, J. García, and E. Mazur, "Bulk heating of transparent materials using a high-repetition-rate femtosecond laser," Appl. Phys. A 76, 351-354 (2003).

12. S. M. Eaton, H. Zhang, P. Herman, F. Yoshino, L. Shah, J. Bovatsek, and A. Arai, "Heat accumulation effects in femtosecond laser-written waveguides with variable repetition rate," Opt. Express 13, 4708-4716 (2005).

13. W. Gawelda, D. Puerto, J. Siegel, A. Ferrer, A. Ruiz De La Cruz, H. Fernández, and J. Solis, "Ultrafast imaging of transient electronic plasmas produced in conditions of fem- 
tosecond waveguide writing in dielectrics," Appl. Phys. Lett. 93, 121109 (2008).

14. C. Hnatovsky, R. S. Taylor, E. Simova, V. R. Bhardwaj, D. M. Rayner, and P. B. Corkum, "High-resolution study of photoinduced modification in fused silica produced by a tightly focused femtosecond laser beam in the presence of aberrations," J. Appl. Phys. 98, 013517 (2005).

15. Q. Sun, H. Jiang, Y. Liu, Y. Zhou, H. Yang, and Q. Gong, "Effect of spherical aberration on the propagation of a tightly focused femtosecond laser pulse inside fused silica," J. Opt. A, Pure Appl. Opt. 7, 655-659 (2005).

16. R. Osellame, S. Taccheo, M. Marangoni, R. Ramponi, P. Laporta, D. Polli, S. De Silvestri, and G. Cerullo, "Femtosecond writing of active optical waveguides with astigmatically shaped beams," J. Opt. Soc. Am. B 20, 1559-1567 (2003).

17. V. Diez-Blanco, J. Siegel, A. Ferrer, A. Ruiz De La Cruz, and J. Solis, "Deep subsurface waveguides with circular cross section produced by femtosecond laser writing," Appl. Phys. Lett. 91, 051104 (2007).

18. M. Ams, G. D. Marshall, D. J. Spence, and M. J. Withford, "Slit beam shaping method for femtosecond laser directwrite fabrication of symmetric waveguides in bulk glasses," Opt. Express 13, 5676-5681 (2005).

19. D. Rayner, A. Naumov, and P. Corkum, "Ultrashort pulse nonlinear optical absorption in transparent media," Opt. Express 13, 3208-3217 (2005).

20. A. Dubietis, G. Tamosauskas, G. Fibich, and B. Ilan, "Multiple filamentation induced by input-beam ellipticity," Opt. Lett. 29, 1126-1128 (2004).

21. Y. Nasu, M. Kohtoku, and Y. Hibino, "Low-loss waveguides written with a femtosecond laser for flexible interconnection in a planar light-wave circuit," Opt. Lett. 30, 723-725 (2005).

22. N. D. Psaila, R. R. Thomson, H. T. Bookey, A. K. Kar, N. Chiodo, R. Osellame, G. Cerullo, A. Jha, and S. Shen, "Er:Yb-doped oxyfluoride silicate glass waveguide amplifier fabricated using femtosecond laser inscription," Appl. Phys. Lett. 90, 131102 (2007)
23. Y. Cheng, K. Sugioka, K. Midorikawa, M. Masuda, K. Toyoda, M. Kawachi, and K. Shihoyama, "Control of the cross-sectional shape of a hollow microchannel embedded in photostructurable glass by use of a femtosecond laser," Opt. Lett. 28, 55-57 (2003).

24. M. Ams, G. D. Marshall, and M. J. Withford, "Study of the influence of femtosecond laser polarisation on direct writing of waveguides," Opt. Express 14, 13158-13163 (2006).

25. C. Hnatovsky, R. Taylor, E. Simova, P. Rajeev, D. Rayner, V. Bhardwaj, and P. Corkum, "Fabrication of microchannels in glass using focused femtosecond laser radiation and selective chemical etching," Appl. Phys. A 84, 47-61 (2006).

26. Y. Shimotsuma, P. Kazansky, J. Qiu, and K. Hirao, "Selforganized nanogratings in glass irradiated by ultrashort light pulses," Phys. Rev. Lett. 91, 247405 (2003).

27. A. Ferrer, V. Diez-Blanco, A. Ruiz, J. Siegel, and J. Solis, "Deep subsurface optical waveguides produced by direct writing with femtosecond laser pulses in fused silica and phosphate glass," Appl. Surf. Sci. 254, 1121-1125 (2007).

28. A. Brodeur and S. L. Chin, "Ultrafast white-light continuum generation and self-focusing in transparent condensed media," J. Opt. Soc. Am. B 16, 637-650 (1999).

29. A. Brodeur and S. Chin, "Band-gap dependence of the ultrafast white-light continuum," Phys. Rev. Lett. 80, 44064409 (1998).

30. S. Mao, F. Quéré, S. Guizard, X. Mao, R. Russo, G. Petite, and P. Martin, "Dynamics of femtosecond laser interactions with dielectrics," Appl. Phys. A 79, 1695-1709 (2004).

31. D. Homoelle, S. Wielandy, A. L. Gaeta, N. F. Borrelli, and C. Smith, "Infrared photosensitivity in silica glasses exposed to femtosecond laser pulses," Opt. Lett. 24, 1311-1313 (1999).

32. A. Mairaj, H. Rutt, and D. Hewak, "Fabrication and characterization of continuous wave direct UV $(\lambda=244 \mathrm{~nm})$ written channel waveguides in chalcogenide (Ga:La:S) glass," J. Lightwave Technol. 20, 1578-1584 (2002). 